\title{
Etude expérimentale de l'effet de groupe et de la densité du massif de sol sur les courbes de réaction P.Y des pieux chargés latéralement
}

\author{
S. Mezazigh*, D. Levacher, ${ }^{* *}$, A. Dana*** \\ * Laboratoire de Mécanique, Université du Havre, \\ ** Centre de morphologie, Université de Caen, \\ ** * Université de Jakarta, Indonésie.
}

Résumé : La plupart des recherches sur les pieux chargés latéralement ont été menées sur des pieux isolés alors que ces derniers sont fréquemment utilisés en groupe. Le problème est tridimensionnel et l'interaction sol-pieu est trop complexe pour que cette étude puisse être conduite à l'aide de méthodes théoriques ou numériques. Compte tenu du nombre d'essais nécessaires, elle ne peut guère être réalisée à l'aide d'essais sur des pieux réels. L'article présente une étude expérimentale, sur des modèles réduits centrifugés, du comportement d'un couple de pieux dans un massif sableux. L'analyse est portée simultanément sur les courbes de chargement, des moments de flexion et les courbes de réaction P-Y. Le but principal est la mise en évidence de l'effet de groupe et de la densité sur les courbe $\mathrm{P}-\mathrm{Y}$, pour des pieux installés par battage dans un massif sableux.

\begin{abstract}
The investigations and researches on laterally loaded piles are concerning single piles, but piles are commonly used in groups. The problem is very complex to be study with theoretical or numerical methods. As a large number of tests are necessary, they could not to be run on true scale piles. However, with small scale model tests, it is important to make the simulation as perfectly as possible, either in reversible domain or in rupture domain. The mass forces simulations by centrifuge is well adapted to answer to this kind of problem. All tests have been done at the Laboratoire des Ponts et Chaussées of Nantes to determine the group, the installation and the density effect on P-Y reaction curves of the laterally loaded pile driven into sand. In this paper, the analysis results tests of laterally loaded couples of piles in centrifuge are presented and discussed.
\end{abstract}

\section{Introduction}

Malgré le nombre de publications relatant les travaux sur l'effet de groupe, aucune méthode d'application pratique n'a encore été dégagée.

Les approches théoriques conduisent à des résultats souvent éloignés des données expérimentales. Dans le cas des méthodes élastiques, les interactions entre pieux sont très mal rendues. Ainsi la non symétrie des efforts observée lors des essais n'est pas donnée par les calculs élastiques.

La résolution numérique par éléments finis au moyen de modèles tridimensionnels est capable de pendre en compte la non linéarité du sol ainsi que son hétérogénéité et de donner des informations sur les déplacements, les moments fléchissants le long des pieux et les courbes de réaction du sol. L'effet de groupe peut également être mis en évidence par ce type d'analyse (BROWN et 
col., 1990), le coût des calculs devient alors vite important ce qui limite les possibilités de variations des paramètres. D'autre part, les réponses sont étroitement liées aux modèles rhéologiques introduits pour le sol et pour les interfaces sol-pieu; des calages sont encore nécessaires.

Par ailleurs, un des paramètres importants qui n'est pas pris en considération et qui entraîne des difficultés dans la prévision du comportement d'un groupe de pieux sollicité latéralement, est le remaniement du sol induit lors de la mise en place du groupe, plus particulièrement s'il s'agit de pieux refoulant le sol. En effet les travaux de L.M. JOSÉ et col.(1991) utilisant une analyse simplifiée d'un problème axisymétrique, ont montré l'influence de l'installation des pieux en groupe dans un sable, sur les déplacements et les contraintes horizontales dans le sol. L'auteur recommande alors la valeur $r_{\infty}$ égale à $15 R$ $\left(r_{\infty} \approx 8 \mathrm{~B}\right)$ où $\mathrm{R}$ est le rayon du pieu, comme distance minimum au-delà de laquelle l'interaction entre les pieux du groupe peut être négligée. D'autres auteurs se sont aussi intéressés au problème d'installation de pieux isolés et de groupe de pieux et on montré l'importance de la perturbation résultant de la mise en place (COOKE et col.; 1979; SHUBLAQ, 1992; POULOS, 1994). En général, la mise en place d'un ensemble de pieux modifie les propriétés du sol de façon différente de celle d'un pieu isolé dont le comportement sert de référence. L'effet de groupe peut, suivant les cas, améliorer ou réduire les qualités mécaniques du sol environnant et, par conséquent, conduire à un accroissement ou à une diminution de la charge nominale du pieu en groupe par rapport à celle du pieu isolé.

Les essais sur modèles réduits centrifugés présentent les mêmes avantages que les essais sur modèles de laboratoire (répétitivité, contrôle rigoureux des conditions expérimentales, études paramétriques possibles, étalonnage de la réponse des pieux instrumentés avant essai, .....)

En outre, du fait du respect de certaines conditions de similitude et en particulier des forces de masses, leurs résultats peuvent être transposés aux ouvrages en vraie grandeur, ce qui n'est pas le cas des modèles de laboratoire (GARNIER, 1995).

Le concept de coefficient de réduction pouvant être appliqué aux courbe $P-Y$ du pieu isolé pour prendre en compte l'effet de groupe a été évoqué par 2 ou 3 auteurs. Aucune étude systématique de l'effet de groupe sur les courbes $P-Y$ des différents pieux n'a cependant été entreprise. La raison en est sans doute la difficulté d'obtenir expérimentalement ces courbes de réaction.

\section{Présentation des essais centrifugés}

L'étude bibliographique (Mezazigh, 1995) a montré que les recherches sur l'effet de groupe sont nombreuses. A ce jour, ces travaux n'ont pas encore permis d'élaborer des méthodes pratiques de calcul des groupes à partir des courbes de réaction $\mathrm{P}-\mathrm{Y}$. Celles qui figurent dans les règles actuelles n'ont pas de justification (Fascicule 62).

Sur ces bases, une étude a été entreprise au L.C.P.C (Mezazigh, 1995), l'objectif final étant de fournir aux praticiens des coefficients simples, à appliquer aux courbes de réaction obtenues pour un pieu isolé pour retrouver celles des pieux disposés en groupe. Les résultats présentés dans cet article, concernent 
l'étude de l'effet d'installation par battage et de la densité du massif de sable sur les courbes de réaction.

\subsection{Implantation et mode d'installation des pieux dans le massif sableux} 2.1.1 Instrumentation des pieux

Les pieux utilisés dans cette campagne d'essais sont des tubes en aluminium AU4G, de longueur totale de $380 \mathrm{~mm}$, d'un diamètre externe de 18 $\mathrm{mm}$ et d'une épaisseur de $1 \mathrm{~mm}$. La fiche dans le sol est fixée à $300 \mathrm{~mm}$. Les deux pieux ont été équipés identiquement par 20 paires de jauges de déformation régulièrement espacées de $15 \mathrm{~mm}$. Les étalonnages ont montré que l'instrumentation permettait de déterminer les moments de flexion à $0.1 \%$ près.

Les essais ont été conduits à une accélération centrifuge de $40 \mathrm{~g}$. Ceci permet donc de simuler des pieux prototypes de $0.720 \mathrm{~m}$ de diamètre externe et de $12 \mathrm{~m}$ de fiche. La rigidité dans ce cas est de $514 \mathrm{MN} \cdot \mathrm{m}^{2}$.

Chaque pieu du couple a été instrumenté en tête par deux capteurs de déplacement inductifs de type HBM de course $\pm 10 \mathrm{~mm}$. L'application de l'effort latéral est obtenu à l'aide du dispositif de chargement développé au LCPC et est transmis aux pieux grâce à un câble métallique passant par une poulie et traversant les deux pieux comme le montre la figure 1. Cette liaison par câble permet d'éviter d'introduire des moments de flexion parasites en tête des pieux comme cela a été relaté dans les travaux de WILLIAMS (1979) et RANDOLPH (1981).

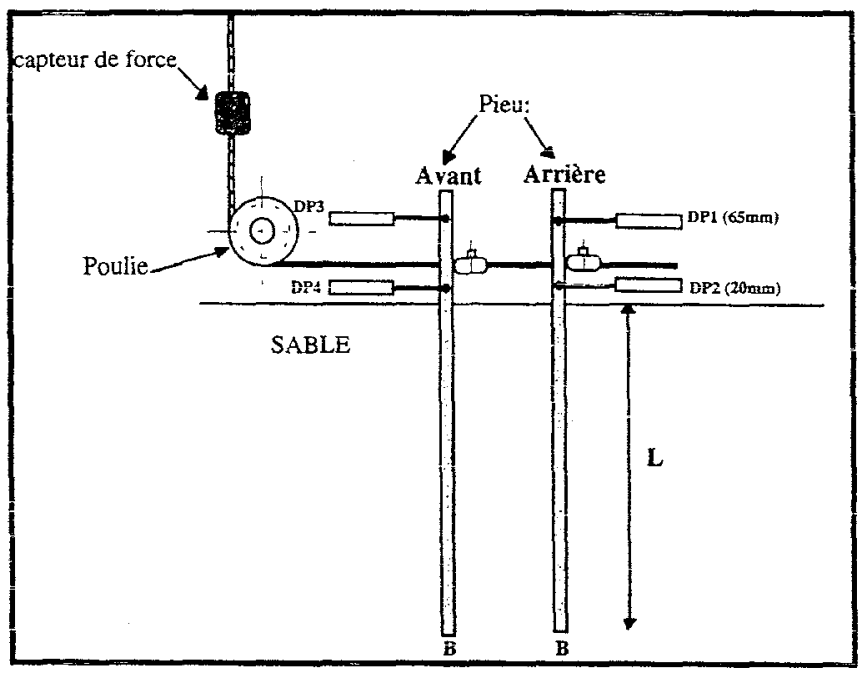

Figure 1: Notation et instrumentation des pieux.

L'effort est appliqué par palier constant jusqu'à la charge maximale, suivant ainsi un mode opératoire classique en France (pieux, ancrages, fondations superficielles). Les précisions de mesure des capteurs de déplacement sont de $\pm 0.02 \mathrm{~mm}$ et celles du capteur de force sont de $\pm 2 \mathrm{~N}$ (valeurs modèle). Les charges appliquées en tête ont été choisies telle que les déplacements restent inférieurs à $0.2 \mathrm{~B}$ (pieux en condition de service). 


\subsubsection{Le massif de sol}

Le matériau utilisé est le sable de Fontainebleau sec. C'est un sable dont environ $70 \%$ des éléments ont un diamètre inférieur à $200 \mu \mathrm{m}$. Pour le modèle de pieu retenu, le rapport entre le diamètre du pieu et celui des grains est d'environ 90 donc très supérieur à la valeur minimale de 40 habituellement admise pour éviter l'effet d'échelle (OVENSEN; 1979).

Le remplissage des conteneurs $(1200 \times 800 \times 360 \mathrm{~mm})$ d'essais se fait par pluviation dans l'air, à l'aide de la trémie automatique du L.C.P.C. Ce système de remplissage permet de confectionner un massif de sable homogène, avec des écarts sur les densités inférieurs à 1\%.(Garnier, 1993; Mezazigh, 1995 ). Deux poids volumiques différents ont été considérées $\left(16.2 \mathrm{kN} / \mathrm{m}^{3}\right.$ et $\left.15.5 \mathrm{kN} / \mathrm{m}^{3}\right)$ correspondant à des indices de densité de 0.81 et 0.63 .

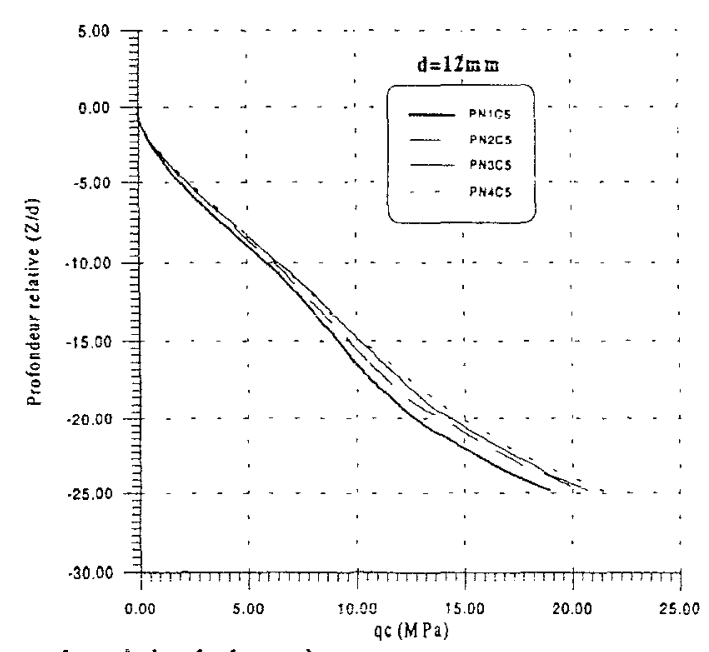

* $\mathrm{d}=$ diamètre du mini-pénétromètre

Figure 2: Mesures pénétrométriques en macrogravité sous accélération de $40 \mathrm{~g}$.

La densité de mise en place est contrôlée a posteriori à l'aide de boîtes calibrées placées au sein du massif lors du remplissage. L'ensemble des résultats des contrôles ont montré une très bonne répétitivité dans la réalisation des massifs de sols. De plus, afin de vérifier la bonne homogénéité verticale du massif de sable, des essais au mini-pénétromètre statique LCPC embarquable en centrifugeuse sont réalisés en cours de centrifugation à $40 \mathrm{~g}$, niveau requis pour les essais. La figure 2 donne un exemple des profils pénétrométriques obtenus au cours des essais.

La compagne d'essais réservée à l'étude de l'effet de groupe a comporté en tout 40 essais dont 18 ont été réservés pour des couples de pieux battus et disposés à différents espacements variant de 2 à $8 \mathrm{~B}$. Les paramètres étudiés sont donc :

- la distance entre les pieux du couple: $2 \mathrm{~B}$ à $8 \mathrm{~B}$,

- le mode de mise en oeuvre (avec ou sans refoulement),

- l'ordre de mise en oeuvre (pour les pieux avec refoulement),

Il est d'abord apparu que l'ordre de mise en place n'avait pas d'effet significatif sur le comportement du couple sous charge latérale. 
Afin d'éviter les effets de bord du conteneur et toute influence des essais entre eux, les pieux ont été disposés tels que la distance entre les bords du conteneur et entre chaque zone d'essai soit supérieure à 10B. Le battage des pieux est effectué à $1 \mathrm{~g}$ à l'aide d'un dispositif de battage spécial développé au L.C.P.C).

Par ailleurs des essais doublés de couples de pieux et ceux des pieux isolés de référence ont été doublés d'un conteneur à l'autre dans le but de confirmer leur répétitivité et la concordance d'un conteneur à l'autre. Les figures 3 donnent un exemple de ces vérifications réalisées sur les pieux de référence. Les résultats obtenus montrent clairement la bonne répétévitité des essais.
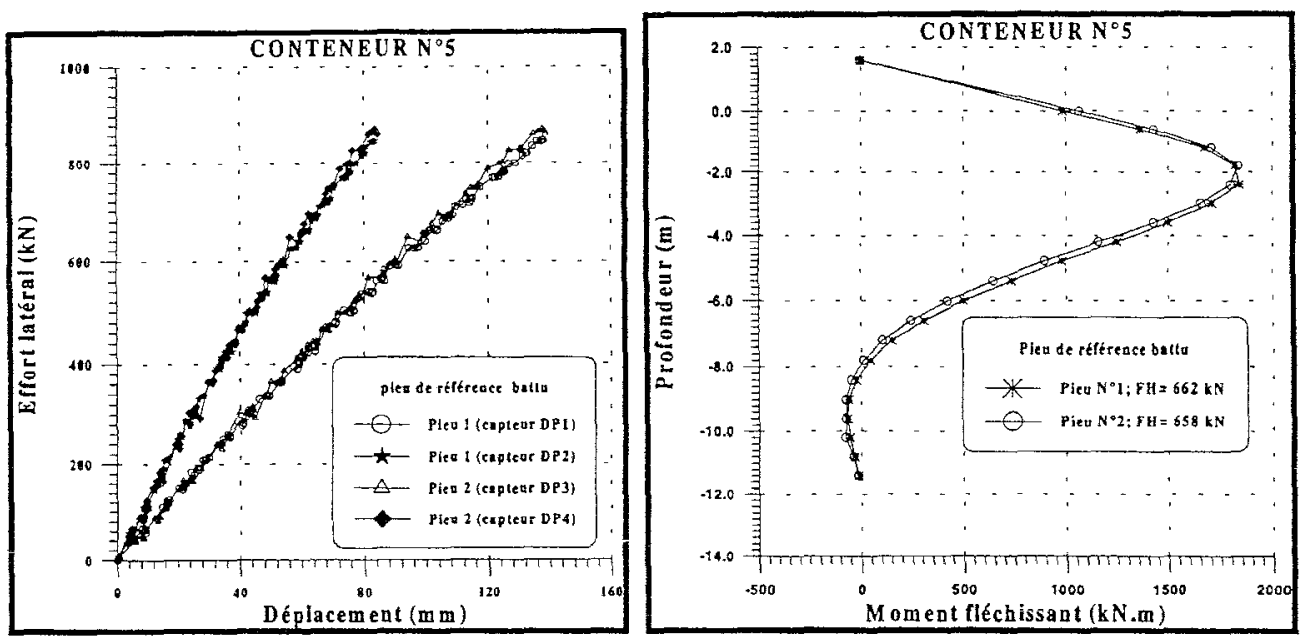

Figure 3: Comparaison des déplacements en tête et des moments fléchissants mesurés sur les pieux de référence 1 et 2 du conteneur $N^{\circ} 5$.

\section{Analyse des résultats des essais sur modèles centrifugés}

\subsection{Influence de l'espacement entre les pieux sur le comportement du groupe} 3.1.1 Déplacements en tête et effort repris par le groupe

Les courbes de chargement obtenues ont montré que, poir ce type de pieux, l'espacement a peu d'influence sur leur comportement (figure 4). Les mêmes constatations ont été faites pour les différents capteurs de déplacement utilisés.

Une méthode simple pour évaluer l'effet de groupe est de calculer l'efficacité du groupe. Ceci revient à comparer la charge latérale moyenne par pieu dans le groupe à la charge latérale pour un pieu isolé pour un même déplacement horizontal.

Pour les pieux étudiés ici, l'efficacité du groupe a été déterminée pour des déplacements inférieurs ou égaux à $10 \%$ du diamètre B des pieux correspondant à des sollicitations de service. Le tableau I regroupe les résultats des valeurs de l'efficacité du groupe (déterminée à partir des valeurs de déplacement enregistrés sur le capteur DP2 situé à $20 \mathrm{~mm}$ par rapport à la surface du sol).

Le calcul des coefficients d'efficacité du groupe effectué dans ce cas confirme que le comportement des pieux du couple est proche de celui du pieu isolé. En effet, les valeurs de ces coefficients sont assez peu différentes de $100 \%$ et ceci indépendamment de l'espacement entre les pieux du groupe, comme le 
montre le tableau I. On remarque cependant que ces coefficients présentent des valeurs parfois légèrement supérieures à 100 . Ceci est probablement dû à la réponse des pieux qui semble gouvernée en priorité par l'état du massif au voisinage de chaque pieu, très affecté par la mise en place. Ces constatations sont valables quelque soit la densité du massif du sol.

On peut cependant conclure que l'effet de groupe global est faible (efficacité $(e)=1$ à $10 \%$ prés) et que le comportement des deux pieux du couple se rapproche de celui d'un pieu isolé. Comme on le verra plus loin, cela ne signifie pas que les deux pieux du couple reprennent les mêmes efforts, égaux à ceux du pieu isolé.
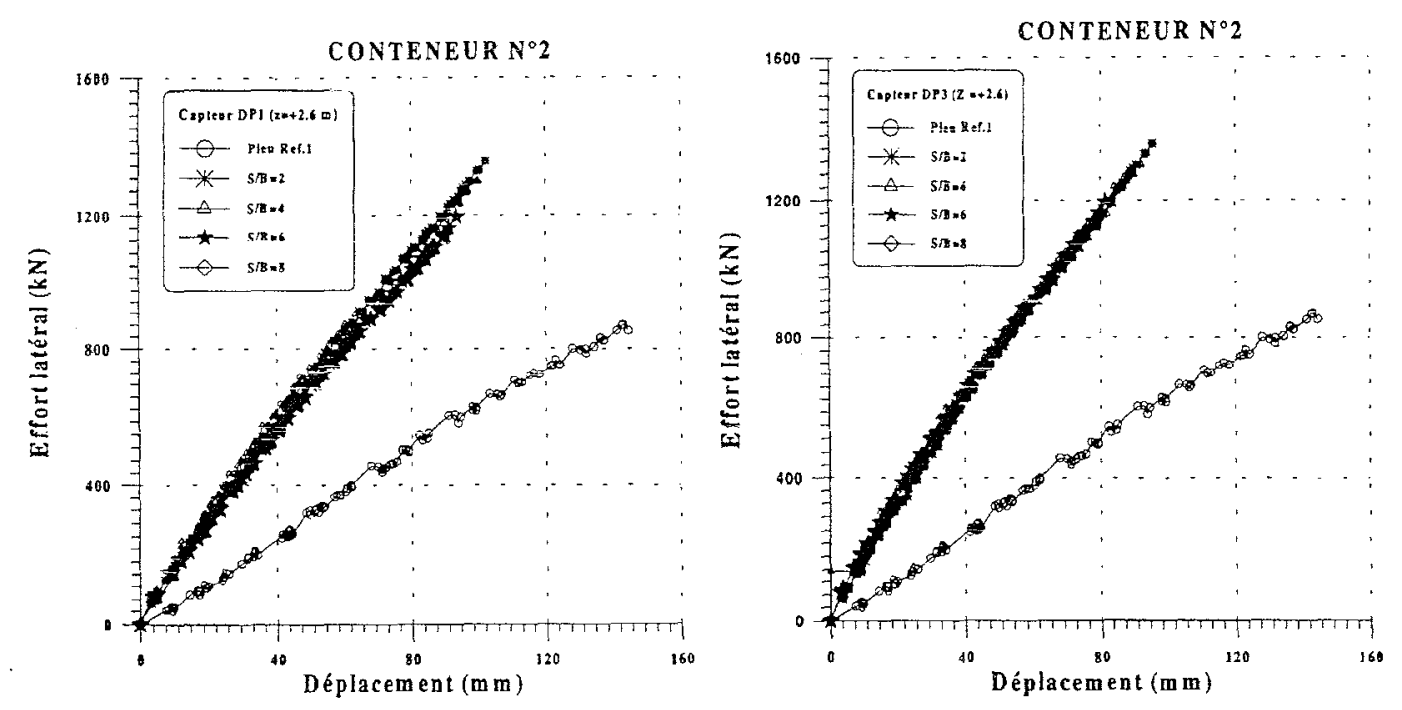

Figure 4: Influence de l'espacement sur le déplacement en tête des pieux battus (Effort total sur les couples et effort sur le pieu isolé).

Tableau I: Efficacité du groupe de pieux battus (valeurs moyennes)

\begin{tabular}{|c|c|}
\hline Espacement relatif S/B & Efficacité du groupe e (\%) \\
\hline 2 & 98.4 \\
\hline 4 & 104.9 \\
\hline 6 & 112.2 \\
\hline 8 & 104.6 \\
\hline
\end{tabular}

\subsubsection{Les moments fléchissant}

Dans le cas des pieux installés par battage à un espacement de $2 \mathrm{~B}$, on constate une légère différence de comportement entre le pieu arrière et le pieu avant comme on peut le voir sur la figure 5. En effet, à cet espacement, on note qu'au niveau de la surface du sol, le pieu avant du couple présente des moments fléchissants environ $20 \%$ fois plus importants que sur le pieu arrière. Le moment maximum étant $12 \%$ plus grand. En outre, le point de moment maximum est situé à une profondeur plus faible sur le pieu avant. Cet effet disparaît rapidement dès l'espacement de 4B comme le montre la figure 6 . On signale par ailleurs, que ces 
observations ont été faites pour tous les incréments de chargement, indépendamment de l'ordre de mise en place des pieux.

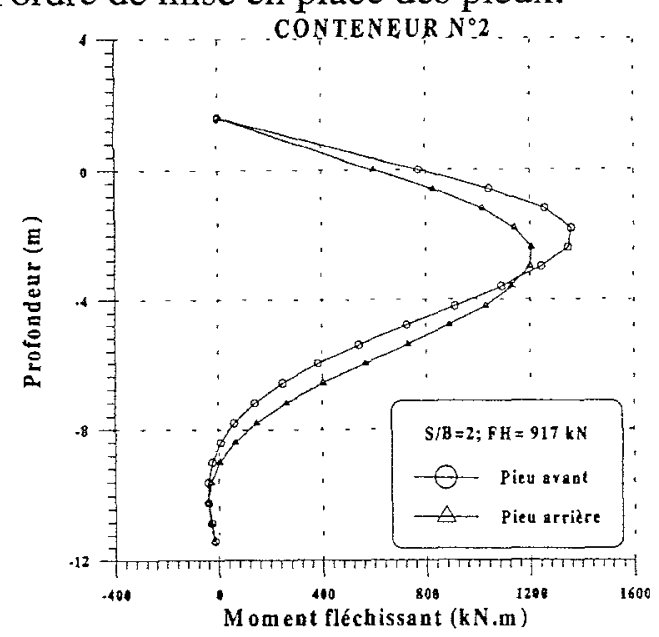

Figure 5: Influence de l'espacement sur le comportement des pieux battus ( $\operatorname{cas} S / B=2$ ).
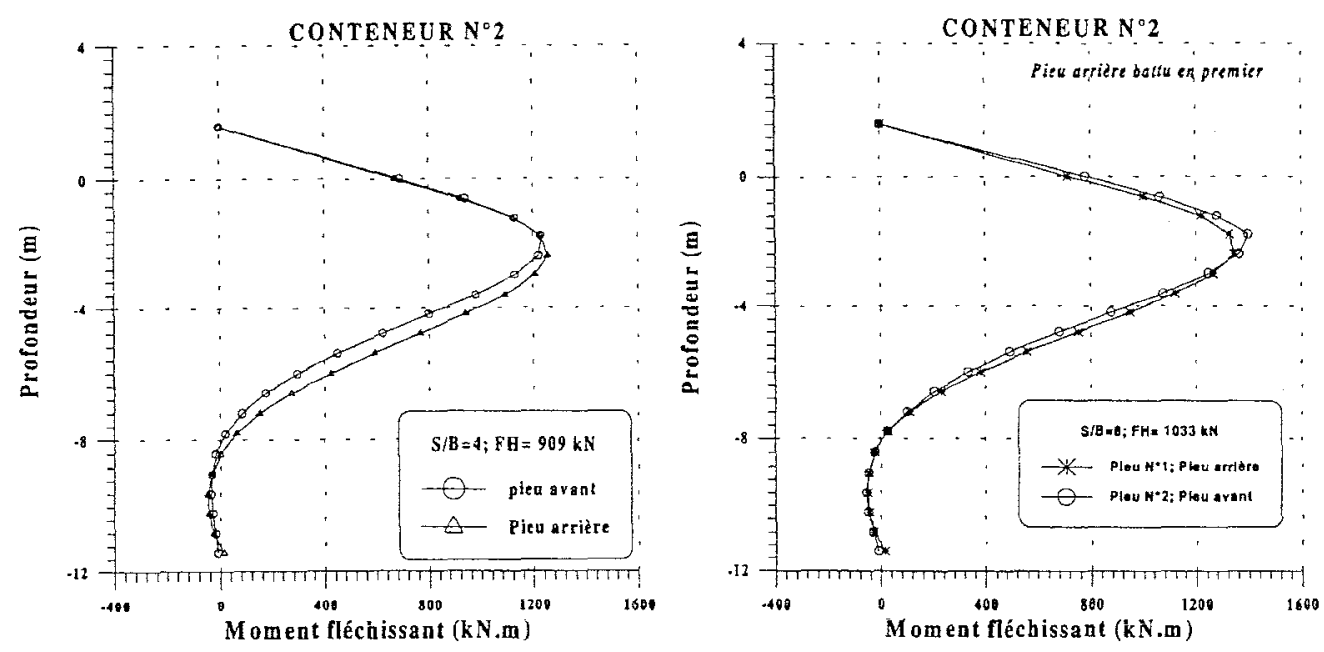

Figure 6: Influence de l'espacement sur le comportement des pieux battus $(S / B=4 ; S / B=8)$.

\subsection{Courbes de réaction $P$-Y}

\subsubsection{Construction des courbes P-Y}

Les mesures expérimentales obtenues par les jauges sont lissées par des fonctions splines quintiques puis font l'objet d'une double intégration et d'une double dérivation pour aboutir aux déplacements et aux pressions et, finalement aux courbes de réaction P-Y. Diverses vérifications sont effectuées pour garantir la validité de ces courbes de réaction : équilibre statique du pieu, calcul à rebours de la réponse du pieu sous charge latérale, concomitance des points de pression nulle et déplacement nul.Les courbes de pression $P(z)$ présentés sur la figure 7 sont donc déterminées par double dérivation des courbes des moments.

$$
P(z)=\frac{d^{2} M(z)}{d z^{2}}
$$



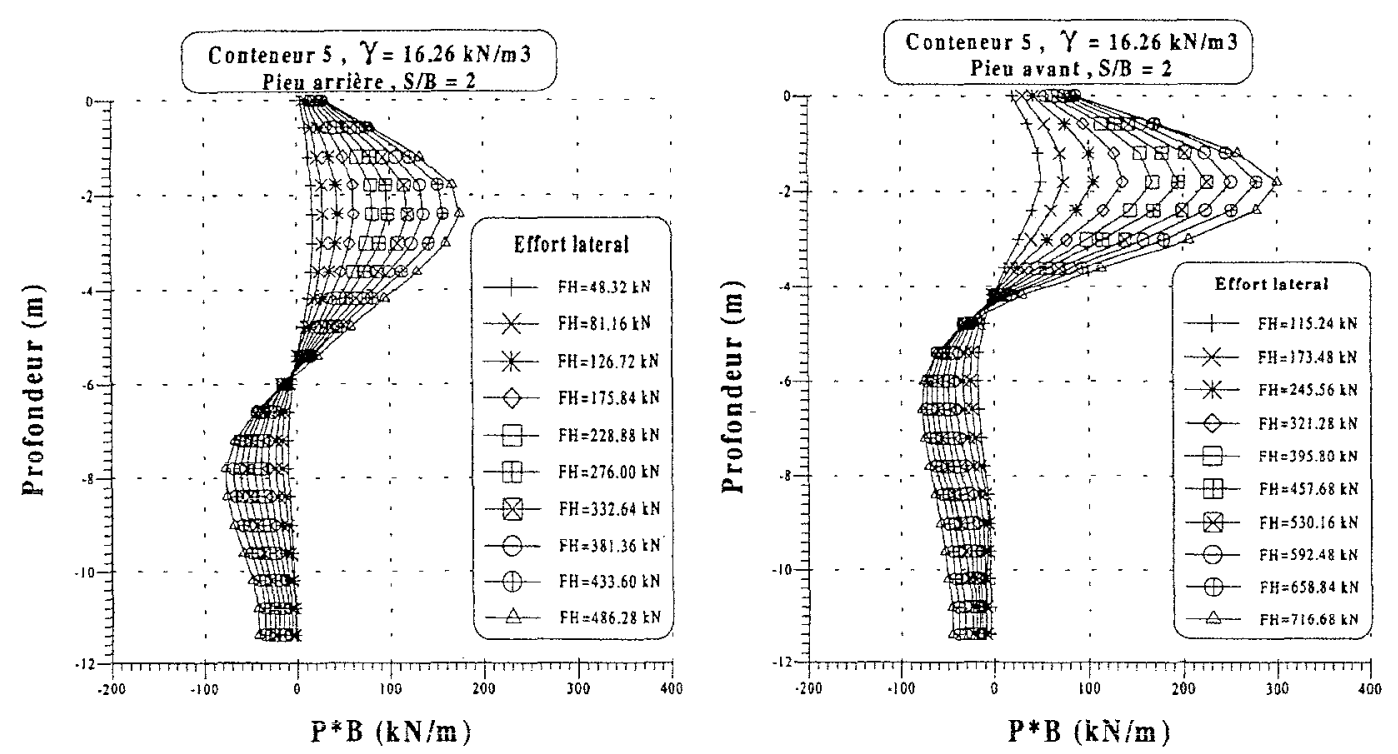

Figure 7: Courbes de pressions obtenues par double intégration des courbes des moments fléchissant (Conteneur 5).
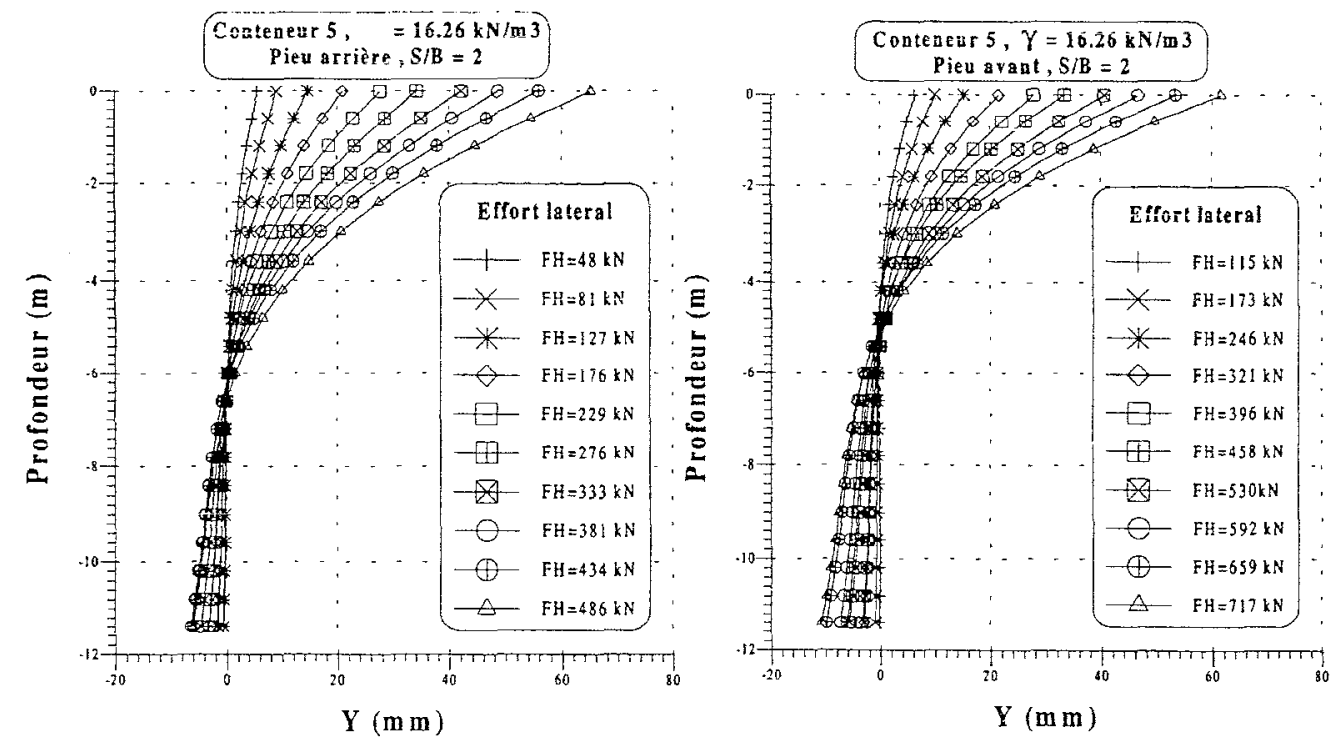

Figure 8: Déformées des pieux obtenues par double intégration des courbes de moments fléchissant (Conteneur 5).

La déformée des pieux $Y(z)$ (figure 8 ) est obtenu par contre par double intégration des courbes de moments fléchissant. Les deux constantes d'intégrations sont fournies par des capteurs de déplacements installés en tête des pieux.

$$
Y(z)=\frac{1}{E I} \iint M(z) \cdot d z \cdot d z+C_{1} \cdot z+C_{2}
$$


Cette opération ne pose aucun de problème particulier et est réalisée par une méthode simple d'intégration numérique des courbes des moments fléchissant. Par contre, la double dérivation, qui permet de déduire les pressions le long des pieux, est une opération délicate puisque on cherche à déterminer la courbure des courbes des moments. Dans notre cas, cette dernière a été réalisée grâce au logiciel SLIVALICS (Deny, 1985) qui repose sur un lissage des courbes par morceaux de polynôme de cinquième dégrée (Spline quintique), Aucune conditions supplémentaire n'est imposée pour l'obtention de ces courbes de pression (pression nulle en tête et en pied pour des pieux dans un massif sableux). Le seule critère utilisée pour l'acceptation des courbes de pressions obtenues est la vérification de l'équilibre statique le long des pieux à $5 \%$ prés.

Pour chaque profondeur, les courbes de réaction $P-Y$ sont ensuite construites point par point à partir des courbes précédentes de déplacements et de pressions. Des constatations de portée générale peuvent être faites sur ces courbes de réaction :

- Les réactions du sol, pour un déplacement donné, croissent avec la profondeur jusqu'à $4 B$ ou $5 B$. Au delà de ces profondeurs, les courbes $P-Y$ semblent se stabiliser ou tout au moins évoluer beaucoup plus lentement. Cette valeur pourrait correspondre à l'apparition d'une profondeur critique (Mezazigh, 1995).

- Plus on s'enfonce en profondeur (au-delà de $5 B$ pour le pieu avant et $6 B$ pour le pieu arrière) on note simultanément un changement de signe des pressions et des déplacements.

\subsubsection{Analyse des courbes P-Y}

\subsubsection{Influence de l'espacement}

D'abord, on peut constater que la position du point de pressions et de déplacements nuls des deux pieux avant et arrière d'une part, et, d'autre part de celle du pieu isolé, n'est pas situé à la même profondeur $(0,50 \mathrm{~L}$ pour le pieu arrière et $0,35 L$ pour le pieu avant dans le cas d'un espacement inférieur à $4 B$ ). Ceci ne permet pas donc de proposer des coefficients de réduction de pressions " $P_{r}$ " et de déplacement " $Y_{r}$ " comme rapport entre les pressions et les déplacement des pieux avants et arrières et ceux du pieu isolé. 


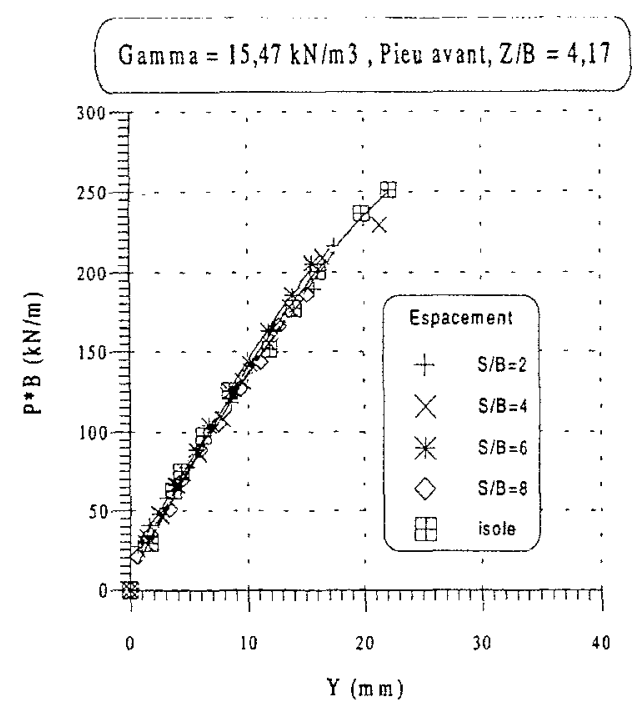

(a)

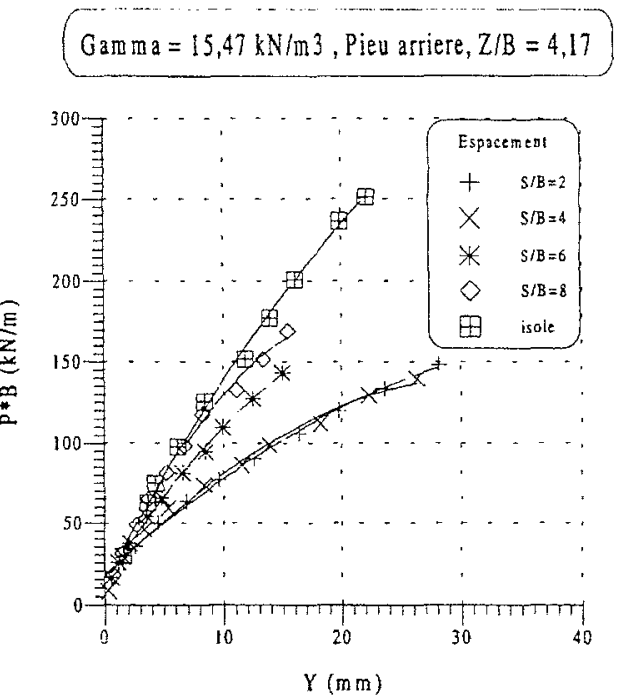

(b)

Figure 9: Comparaison des courbe P-Y du pieu isolé et des pieux avants et arrières des couples pour différents espacements (Conteneur 6).

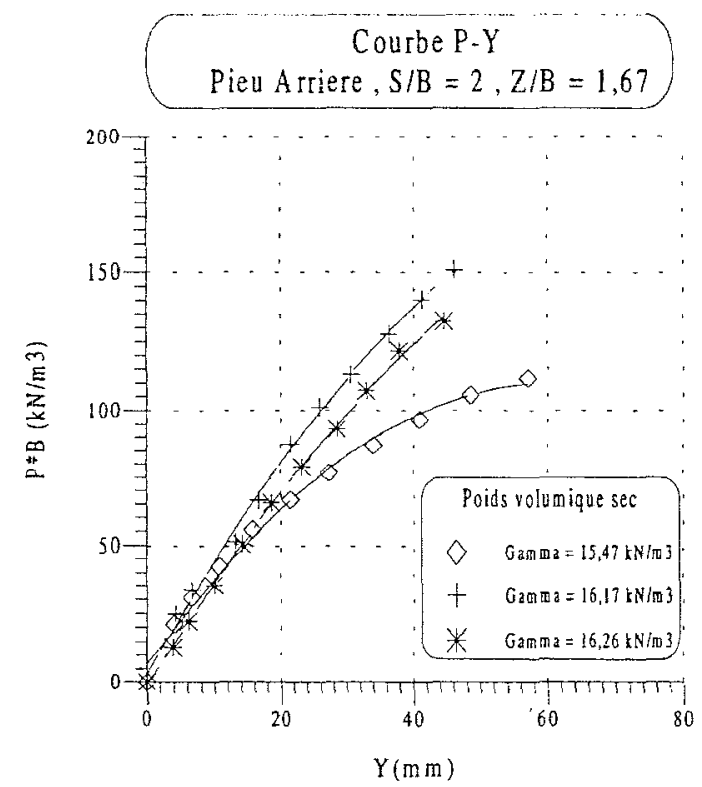

(a)

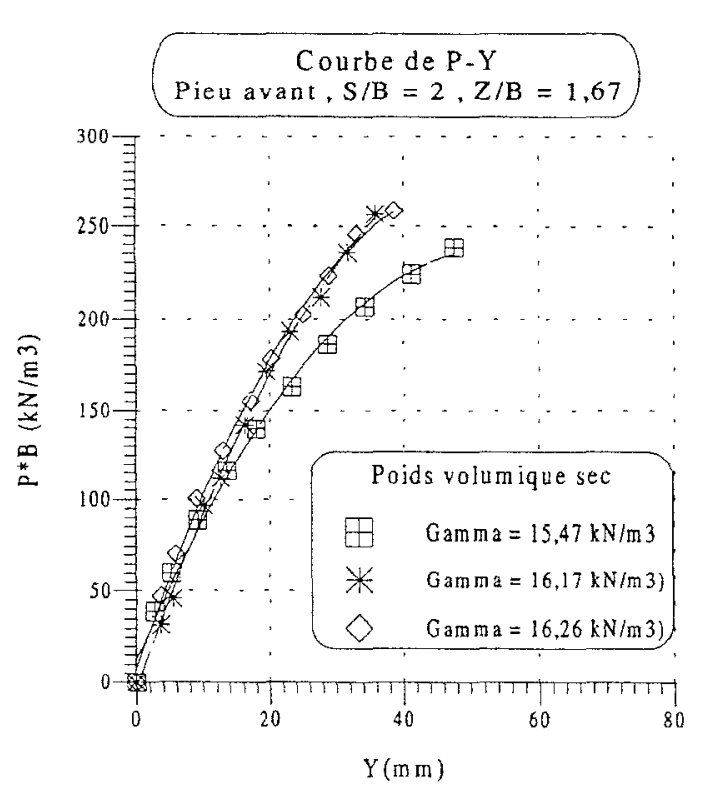

(b)

Figure 10: Courbe $P-Y$ des pieux avant et arrière pour les différents densités de massif sableux.

Cependant, les observations suivantes peuvent être faites sur des courbes $P-Y$ pour des profondeurs inférieures à $5 B$ :

- les courbes $P-Y$ des pieux avant, pour les différents espacements étudiés ont le même comportement qu'un pieu isolé et ceci quel que soit l'espacement relative entre les pieux. Ceci a été constaté pour les différentes profondeurs (figure 9-a).

- Par contre, pour les pieux arrière, un effet de groupe est ressenti mais uniquement pour les espacements inférieurs ou égal à $4 B$, où on a constaté une 
réduction importante du module réaction tangent à l'origine de l'ordre $50 \%$ comme le montre la figure 9-b. Ces constatations ont été observées pour les différentes couches du sol.

\subsubsection{Influence de la densité du massif}

Deux densités du sable ont été considérées : $\gamma_{d}=15.5$ et $16,2 \mathrm{kN} / \mathrm{m}^{3}$. Pour les faibles espacements $(S / B<4)$, l'analyse du comportement des pieux a révélé, au niveau des pieux arrières, une influence relativement faible, de l'ordre $10 \%$, sur les modules tangents à l'origine des courbes de réaction $P-Y$.

Par ailleurs, pour les grands déplacements du pieu arrière, l'effet de la densité est plus ressenti au niveau des pressions et l'écart enregistré pour les deux densité étudiées est d'environ 30\% (figure 10-a).Cette influence s'estompe dès que l'espacement entre les pieux du couple dépasse 4B.

Par contre, pour les pieux avant l'effet de la densité est moins ressenti et l'écart entre les pressions du sol à différentes densités est beaucoup plus faibles et reste inférieur à 5\% (figure 10-b).

\section{Conclusion}

Une étude sur des modèles réduits centrifugés de pieux chargés latéralement $P-Y$ a été effectuée au L.C.P.C de Nantes (MEZAZIGH, 1995). Le but étant la détermination de l'effet de groupe et du mode d'installation des pieux sur les courbes de réactions. Au total 18 essais de pieux battus, réparties sur 3 conteneurs, ont été analysés. Les paramètres essentiels sont :

- Les espacements entre les pieux du groupe (de $S / B=2$ à $S / B=8$ ),

- La densité du massif.

L'analyse des résultats recueillis des courbes expérimentales des moments de flexions, des courbes de chargement et des courbes de réaction nous a révélé essentiellement les points suivants :

- D'abord, le comportement des pieux du groupe battus dans un massif sableux est régis par l'état du massif au voisinage de chaque pieu, très affecté par la mise en place des pieux. L'effet de groupe global est très faible (efficacité de l'ordre de 1 à $10 \%$ prés). Cependant, on peut noter les points imports suivants :

- Pour les faibles espacements $(S / B<4)$ le pieu avant étant un peu plus sollicité que le pieu arrière (pour $S / B=2$, l'effet en tête est $20 \%$ plus fort et le moment maximum est $12 \%$ plus grand),

- Par ailleurs, l'analyse des résultats des essais a révélé que le pieu avant présentait pratiquement le même comportement que le pieu isolé et ce quel que soit l'espacement entre les pieux du groupe,

- Par contre, pour les pieux arrières et surtout pour les faibles espacements $(S / B<4)$, une importante réduction du module tangent à l'origine par rapport au pieu isolé a été observée (de l'ordre de $50 \%$ pour $S / B=2$ ). Cet effet disparaît dès que l'espacement dépasse $4 B$.

En ce qui concerne de l'effet de densité, on a constaté les points suivants : 
- Pour les faibles déplacements ( $<0,5 B$, conditions de service), une influence négligeable de la densité sur le module tangent des courbes $P$ - $Y$ est observée pour les deux pieux du couple,

- Pour les grands déplacements, l'analyse a révélée une forte réduction des pressions au niveau du pieu arrière. Dans notre étude, celle-ci a atteint une valeur de l'ordre de $30 \%$ pour un espacement relative $S / B=2$ et à une profondeur de $Z=1,67 B$. Cet effet s'annule dés qu'on dépasse les espacements de $4 B$,

- Pour le pieu avant cet effet sur les courbes de réaction est pratiquement nulle (inférieur à 5\%).

Cependant pour bien cerner cette influence et quantifier cet effet avec plus de précision sur des paliers plastiques, non visibles sur les courbes P-Y étudiées dans les conditions de service, il serait nécessaire d'effectuer d'autres essais à différentes densités et à des grands déplacements $(\geq 2 B)$.

\section{Références bibliographiques}

[1] Brown D., Morrison C. Reese L. (1988), Lateral (1988), "Laieral load behaviour of pile group in sand", A.S.C.E, vol.114, $\mathrm{N}^{\circ} 11$, pp. 1261-1277.

[2] Bouafia A. (1990), "Modélisation des pieux chargés latéralement en centrifugeuse", Thèse de Doctorat de l'ENSM Nantes - France, 367p.

[3] Cooker R.W., Price G, Tarr K. (1979), "Jacked piles in London Clay: a study of load transfer and settlement under working conditions", Géotechnique 29, N², 113-147.

[4] Dana A., (1998),"Etude expérimentale du comportement de groupes de pieux chargés latéralement dans un massif sableux", Rapport de stage D.E.S.S génie portuaire et Côtier, 47p.

[5] Garnier J., Kus B., Levacher D., (1993), Cartographie des densités de massifs de sable reconstitués par pluviation. $6^{\text {eme }}$ Colloque Franco-Polonais de Mécanique des Sols, Douai, pp.105-112.:

[6] Garnier J. (1995), Modèles réduits en mécanique des sols, A.U.G.C, Ecole Centrale de Nantes, 18 Mai, pp. 21-44.

[7] José L., Sayed M. (1991), Group efficiency of piles driven into sands : A simple approach, Geotechnical Engineering Congress, Vol.I, pp.346-355.

[8] Levacher D. (1989), Groupe de pieux sollicités latéralement: Analyse et réflexions en vue d'une étude paramétrique en centrifugeuse, Rapport interne L.C.P.C, M.E.C. No89.08, 29p.

[9] Levacher D., Mezazigh S., Vasslin E., J. Sevin (1996), Instrumentation et comportement de pieux d'accostage sous faible charges horizontales, IV èmes Joumées Nationales GENIE COTIER- GENIE CIVIL, DINARD , FRANCE, 17 19 AVRIL, pp.379-407.

[10] Mezazigh S. (1990), Comportement d'un couple de pieux sollicité latéralement: Etude paramétrique. Mémoire de D.E.A, E.N.S.M Nantes, 88 p.

[11] Mezazigh S, Levacher D., Garnier J.(1990), Comportement d'un couple de pieux sollicité latéralement, Premières Journées Inter-Universitaires, Génie Côtier, LE HAVRE, pp. 149-160. 
[12] Mezazigh S. (1995), Etude expérimentale des pieux chargés latéralement : Proximité d'un talus et effet de groupe, Mémoire de thèse de doctorat de l'Université de Nantes et de l'Ecole Centrale de Nantes, 217p.

[13] Ovesen N.K (1979). The scaling law relationship. Panel discussion. 7ème E.C.S.M.F.E, Brighton, vol.4, pp.319-323.

[14] Poulos H. G., DAVIS E. H.(1980), Pile fondation analysis and design, John WILEY and Son, 232p.

[15] Poulos H.G (1994), Effect of pile driving on adjacent piles in clay. Canadian Geotechnical Journal, Vol. 31, pp.856-867.

[16] Randolph M. (1981), The response of flexible piles to lateral loading, Canadian Geotechnical Journal, Vol.3.1, N², pp.733-751.

[17] Shublaq E. W.(1992), Soil disturbance sue to installation of model piles and pile groups, SOILS AND FUNDATIONS vol. 32, N 4, p.17-26, Dec. 1992.

[18] Williams D.J. (1979). The behaviour of model piles in dense sand. PhD thesis, University of Cambridge. 